\title{
Fig 6-d-1
}

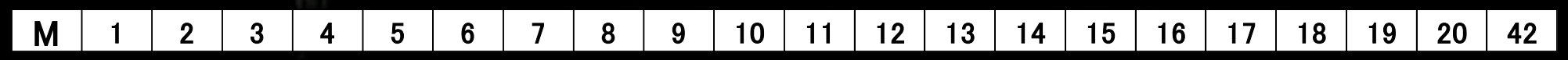

ச - - - - - - 


\section{Fig 6-d-2}

\begin{tabular}{|l|l|l|l|l|l|l|l|l|l|l|l|l|l|l|l|l|l|l|l|l|l|}
$\mathrm{M}$ & 1 & 2 & 3 & 4 & 5 & 6 & 7 & 8 & 9 & 10 & 11 & 12 & 13 & 14 & 15 & 16 & 17 & 18 & 19 & 20 & 42 \\
\hline
\end{tabular}

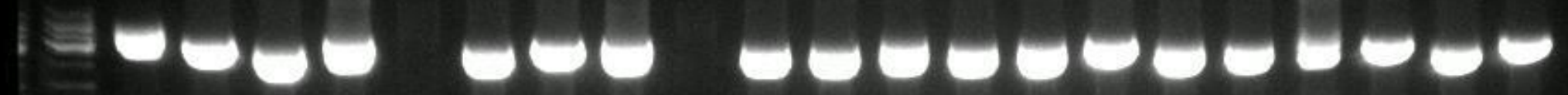

$--------$ 


\section{Fig 6-f-1}

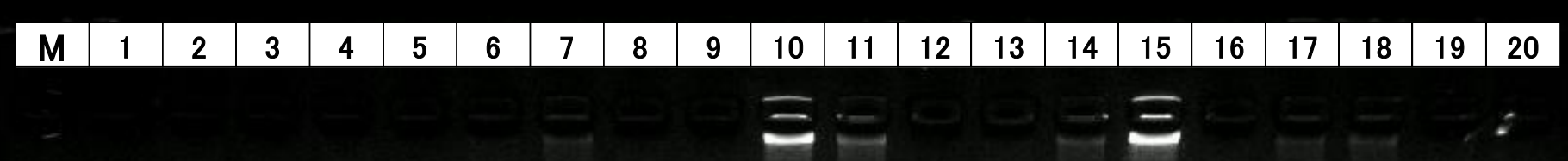

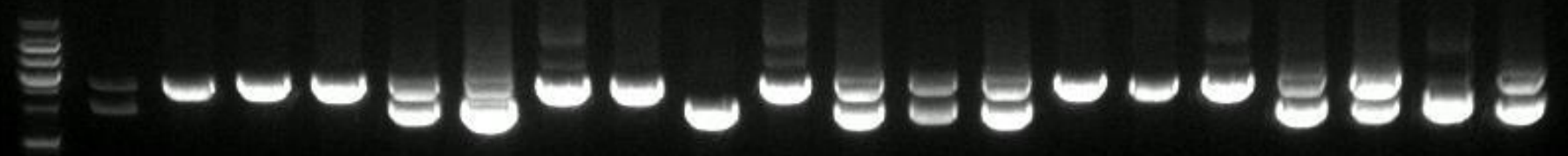
$-$ 


\section{Fig 6-f-1}

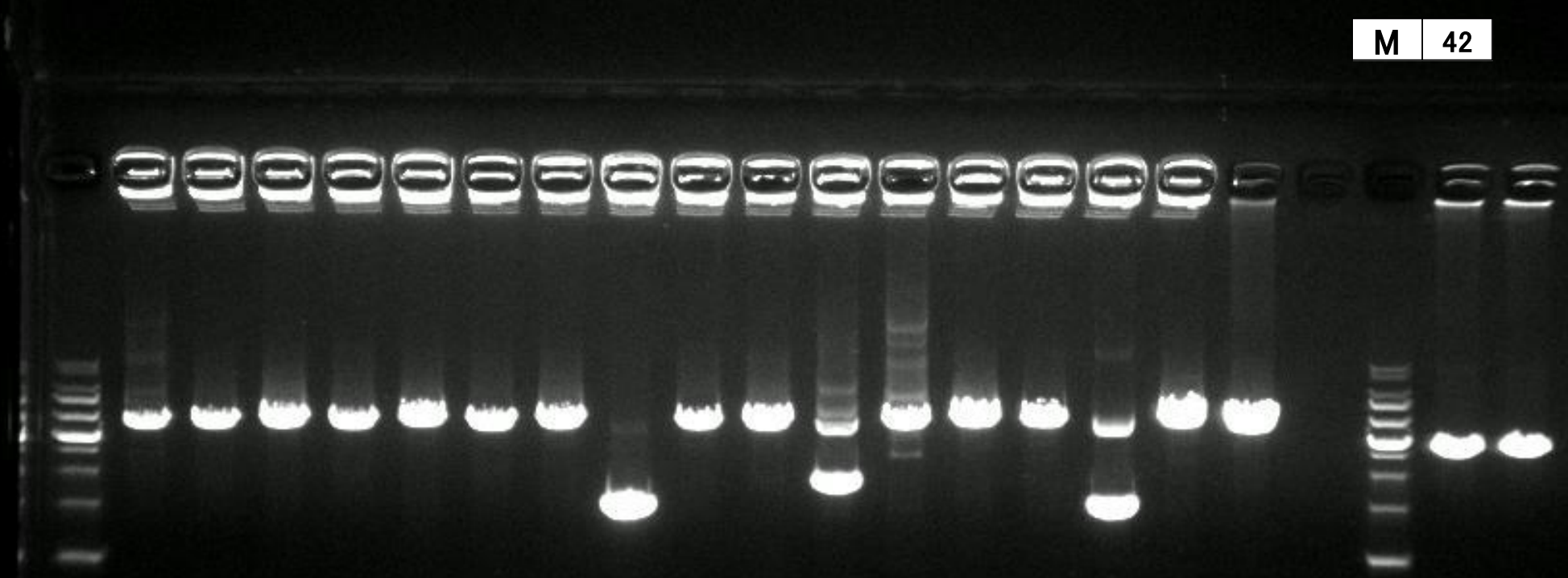




\section{Fig S11-b-1}

\section{\begin{tabular}{|l|l|l|l|l|l|l|l|l|l|l|l|l|l|l|}
\hline $\mathrm{M}$ & 1 & 2 & 3 & 4 & 5 & 6 & 7 & 8 & 9 & 10 & 11 & 12 & 13 & 32 \\
\hline
\end{tabular}}

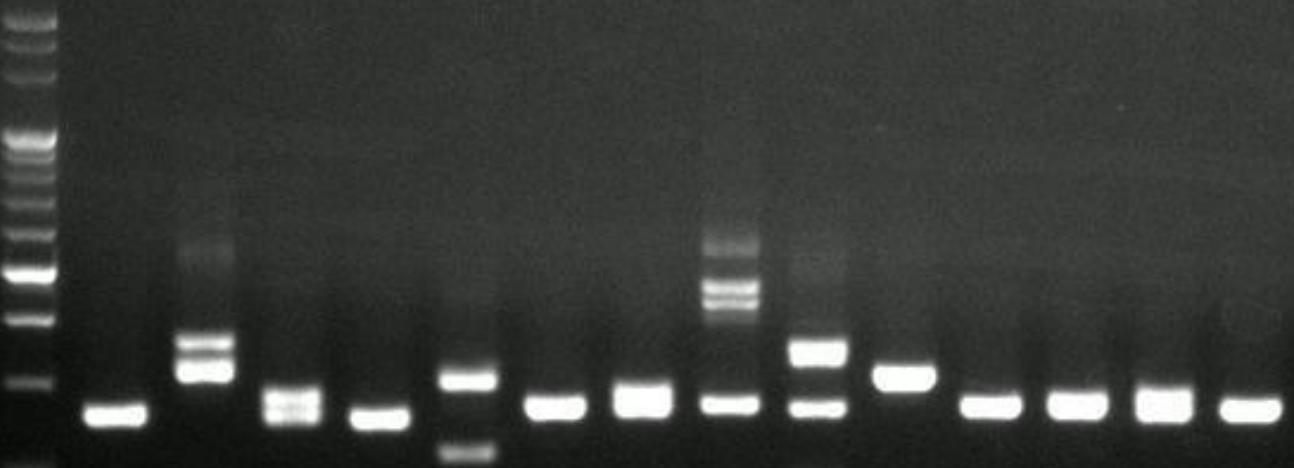




\section{Fig S11-b-2}

\begin{tabular}{|l|l|l|l|l|l|l|l|l|l|l|l|l|l|l|l|l|l|l|l|l|}
\hline $\mathrm{M}$ & 14 & 15 & 16 & 17 & 18 & 19 & 32 & $\mathrm{M}$ & 20 & 21 & 22 & 23 & 24 & 25 & 26 & 27 & 28 & 29 & 30 & 32 \\
\hline
\end{tabular}

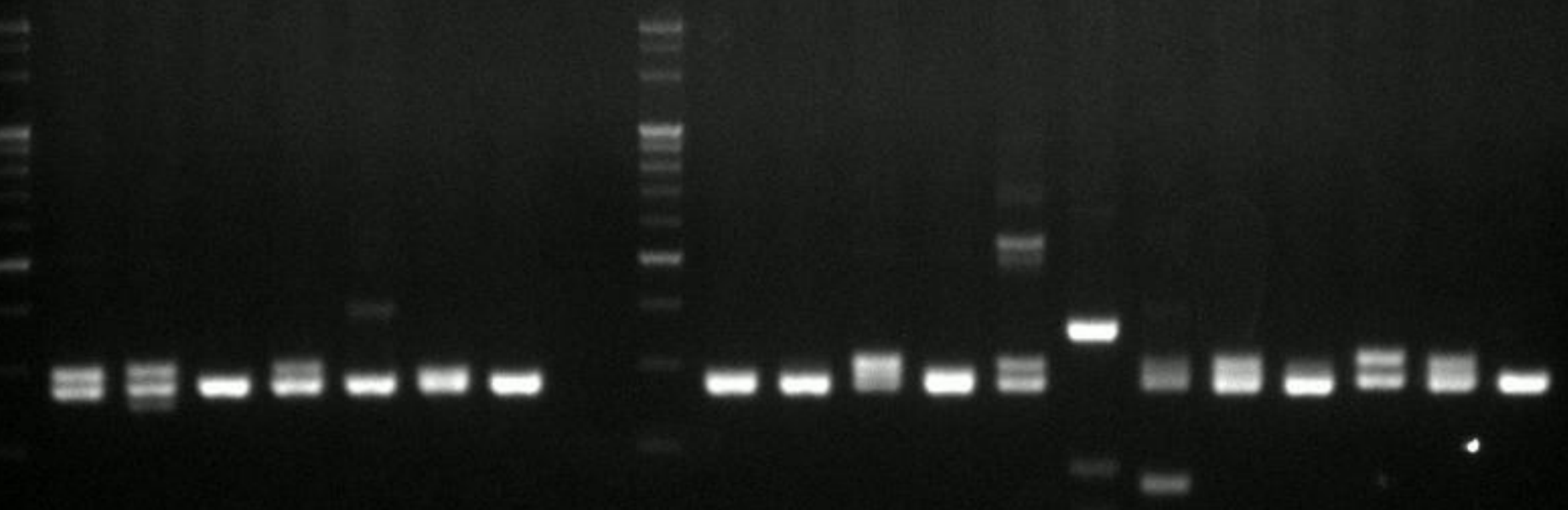




\section{Fig S11-c-1}

\begin{tabular}{|l|l|l|l|l|l|l|l|l|l|l|l|l|l|l|l|l|l|l|l|l|l|l|l|l|}
\hline $\mathrm{M}$ & 1 & 2 & 3 & 4 & 5 & 6 & 7 & 8 & 9 & 10 & 11 & 12 & 13 & 14 & 15 & 16 & 17 & 18 & 19 & 20 & 21 & 22 & 23 & 24 \\
\hline
\end{tabular}

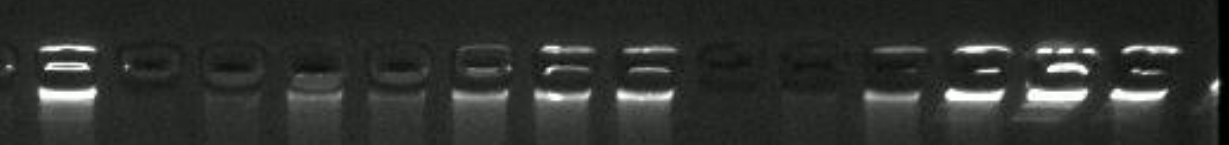

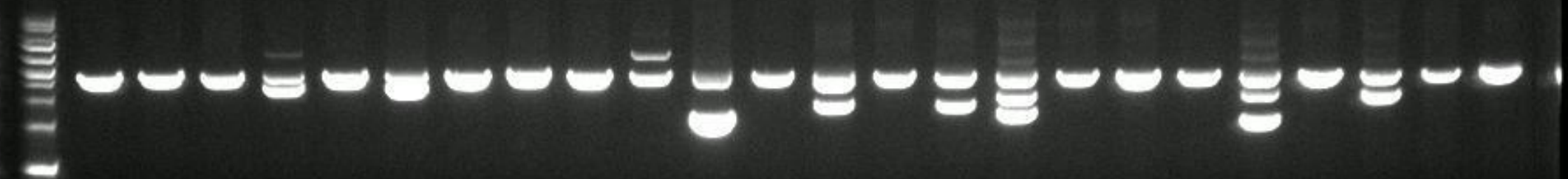




\section{Fig S11-c-2}

\begin{tabular}{l|l|l|l|l|l|l|l|l|}
25 & 26 & 27 & 28 & 29 & 30 & 31 & 32 & $\mathrm{M}$
\end{tabular}

-

$\bullet-\infty \rightarrow \infty$



- 


\section{Fig S12-c}

\section{\begin{tabular}{l|l|l|l|l|}
\hline$M$ & 2 & 10 & Wt & NTC \\
\hline
\end{tabular}}

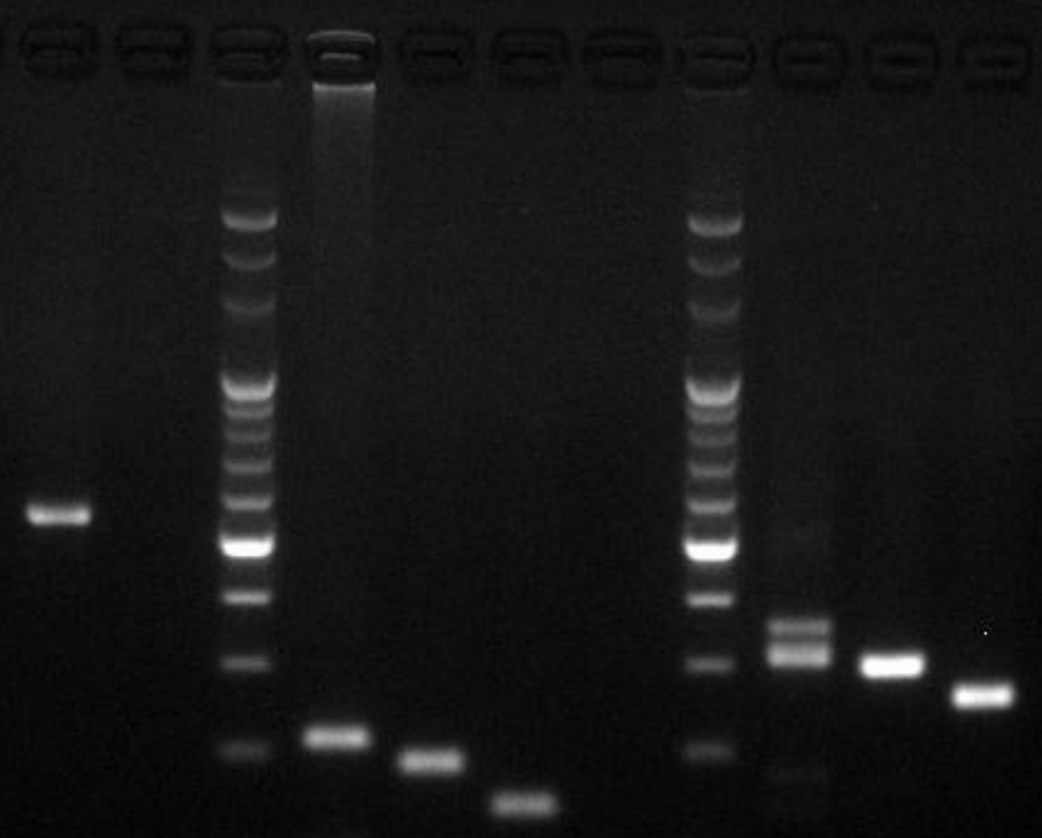




\section{Fig S13-d-1}

\begin{tabular}{|l|l|l|l|l|l|l|l|l|l|l|l|l|l|l|l|l|l|l|l|l|l|l|l|l|}
\hline $\mathrm{M}$ & 1 & 2 & 3 & 4 & 5 & 6 & 7 & 8 & 9 & 10 & 11 & 12 & 13 & 14 & 15 & 16 & 17 & 18 & 19 & 20 & 21 & 22 & 23 & 24 \\
\hline
\end{tabular}

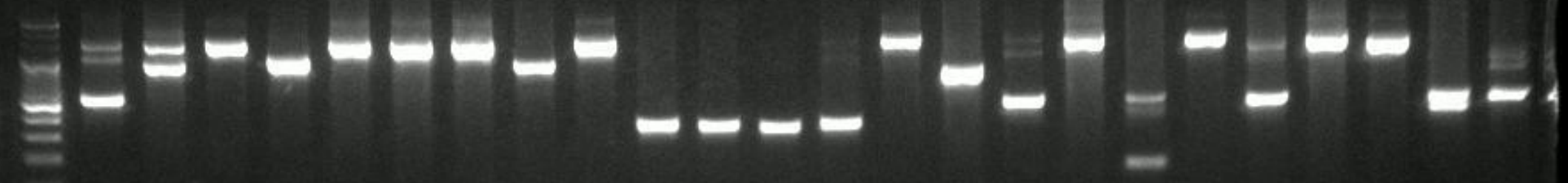

E_-

$\underline{\underline{\underline{E}}}$ 


\section{Fig S13-d-2}

\begin{tabular}{l|l|l|}
\hline 25 & 26 & $\mathrm{M}$ \\
\hline
\end{tabular}

- -

兴- 


\section{Fig S14-d}

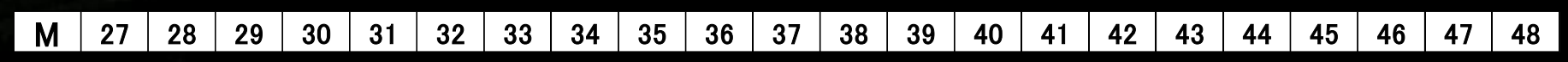

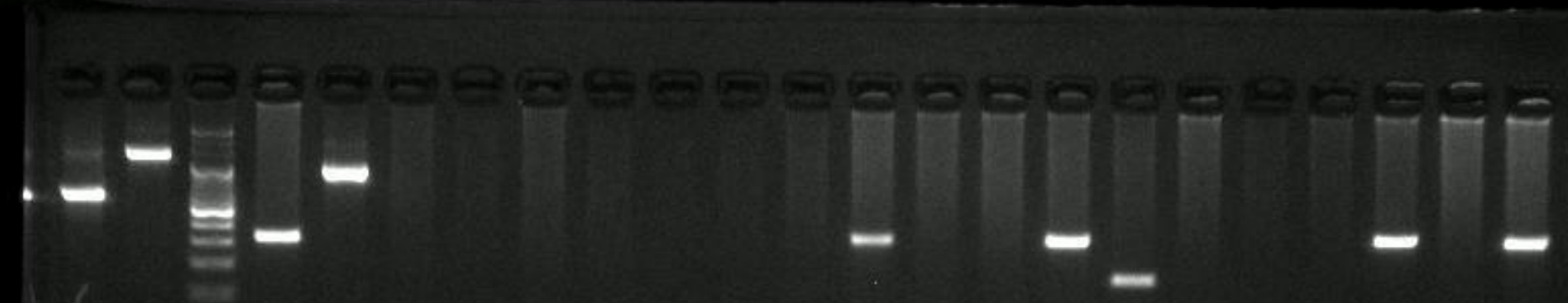

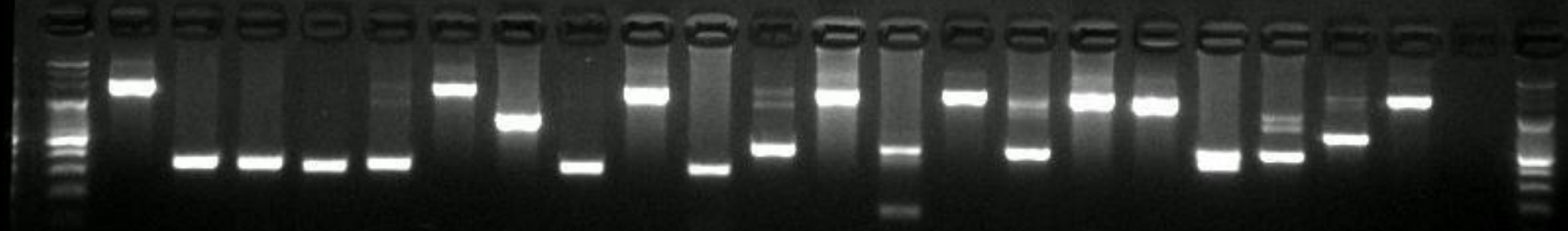




\section{Fig S15-d}

\begin{tabular}{|l|l|l|l|l|l|l|l|l|l|l|l|l|l|l|l|l|l|l|l|l|l|l|l|l|}
\hline $\mathrm{M}$ & 1 & 2 & 3 & 4 & 5 & 6 & 7 & 8 & 9 & 10 & 11 & 12 & 13 & 14 & 15 & 16 & 17 & 18 & 19 & 20 & 21 & 22 & 23 & 24 \\
\hline
\end{tabular}

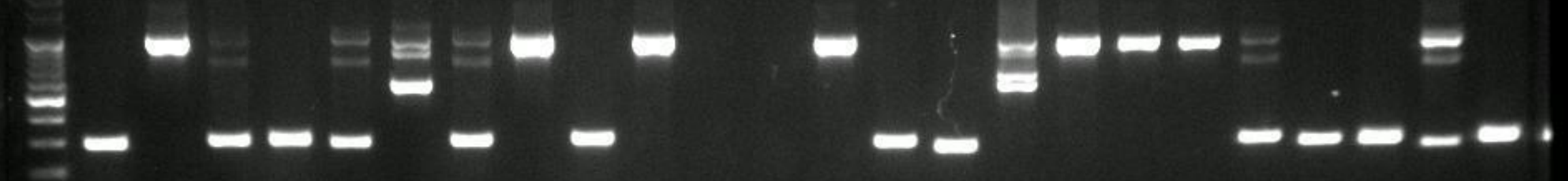

I- 
Fig S15-d-2

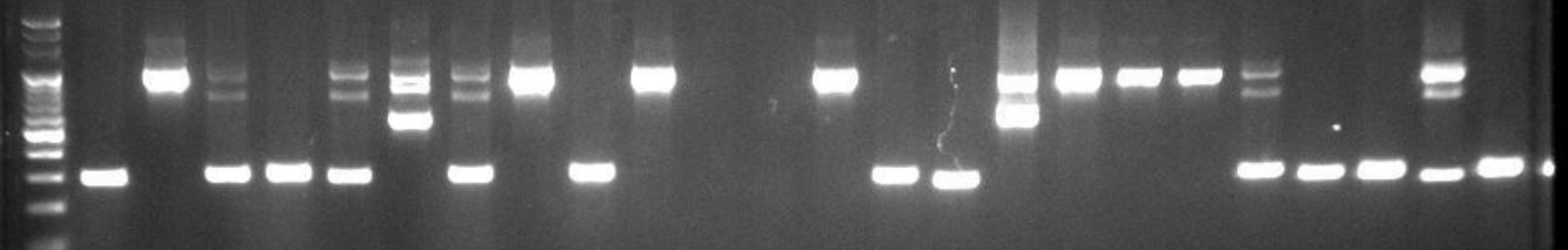

\begin{tabular}{l|l|l|l|l|l|l|l}
\hline M & 25 & 26 & 27 & 28 & 29 & 30 \\
\hline
\end{tabular}

: 


\section{Fig S15-f}

\begin{tabular}{|l|l|l|l|l|l|l|l|}
\hline $\mathrm{M}$ & 8 & 16 & 17 & 1 & 2 & 3 & 30 \\
\hline
\end{tabular}

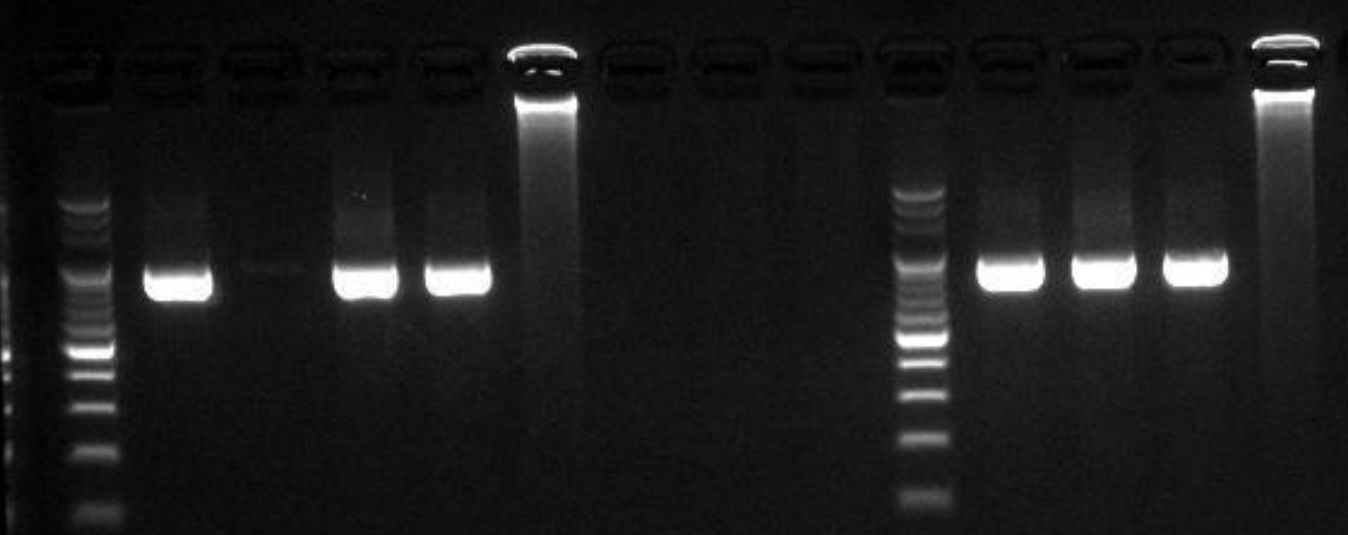




\section{Fig S16-b}

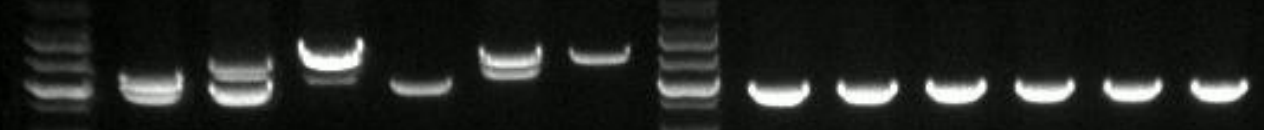




\section{Fig S20-b}

$$
\begin{array}{|l|l|l|l|l|l|l|l|l|l|l|l|l|l|l|l|l|l|l|l|l|l|}
\hline \mathrm{M} & 2 & 5 & 6 & 7 & 10 & 16 & 1 & 14 & 15 & 42 & \mathrm{M} & 2 & 5 & 6 & 7 & 10 & 16 & 1 & 14 & 15 & 42 \\
\hline
\end{array}
$$

シேேேேேே

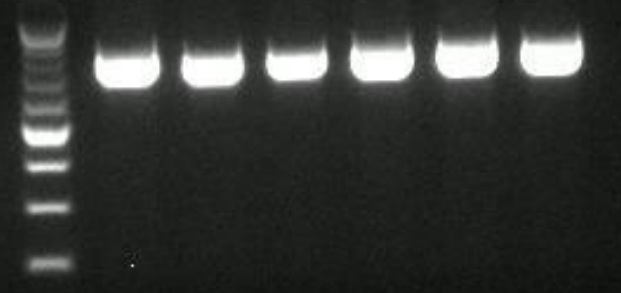


\begin{tabular}{|l|l|l|l|l|l|l|l|l|l|l|l|l|l|l|l|l|l|l|l|l|l|l|l|l|}
\hline $\mathrm{M}$ & 1 & 2 & 3 & 4 & 5 & 6 & 7 & 8 & 9 & 10 & 11 & 12 & 13 & 14 & 15 & 16 & 17 & 18 & 19 & 20 & 21 & 22 & 23 & 24 \\
\hline
\end{tabular}

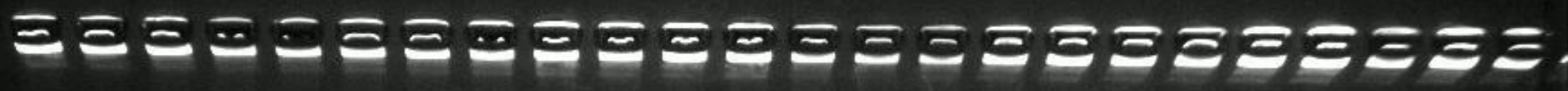

$\equiv$

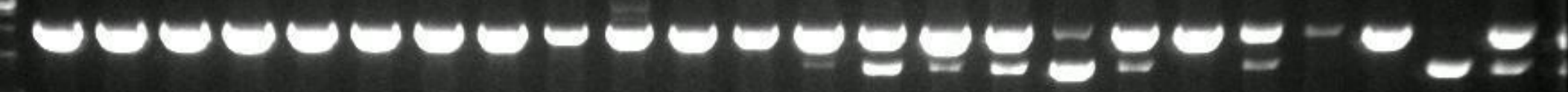
- 
\begin{tabular}{|l|l|l|l|l|l|l|l|l|l|l|l|l|l|l|l|l|l|}
\hline M & 25 & 26 & 27 & 28 & 29 & 30 & 31 & 32 & 33 & 34 & 35 & 36 & 37 & 38 & 39 & 40 & 41 \\
\hline
\end{tabular}

ミこミミミミミミミミミミミミミミミ

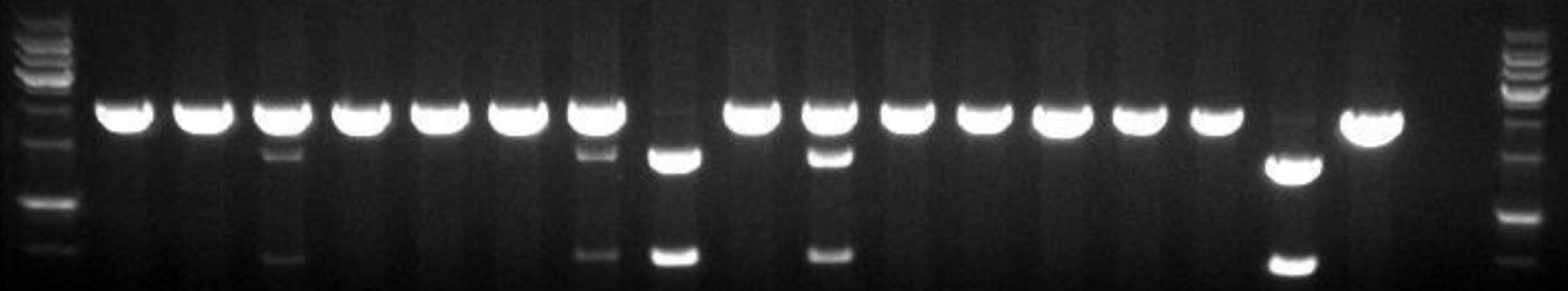




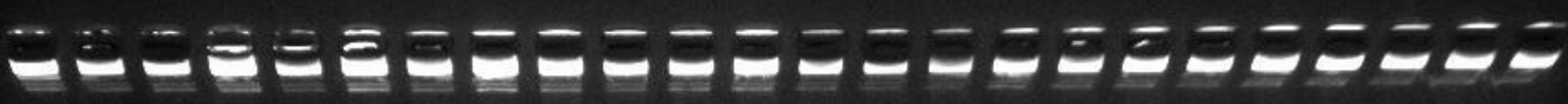

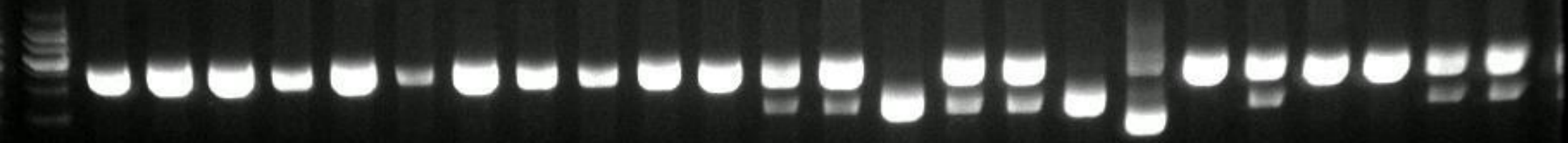
$-$

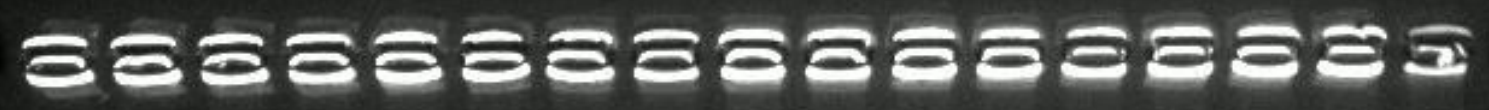

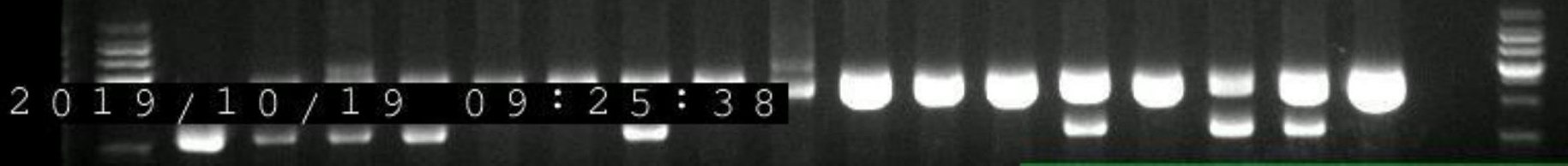


\begin{tabular}{|l|l|l|l|l|l|l|l|l|l|l|l|l|l|l|l|l|l|}
\hline $\mathrm{M}$ & 25 & 26 & 27 & 28 & 29 & 30 & 31 & 32 & 33 & 34 & 35 & 36 & 37 & 38 & 39 & 40 & 41 \\
\hline
\end{tabular}

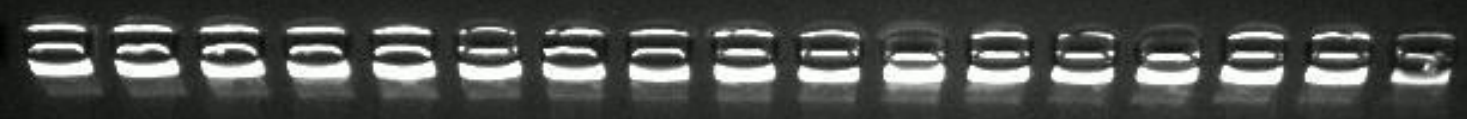

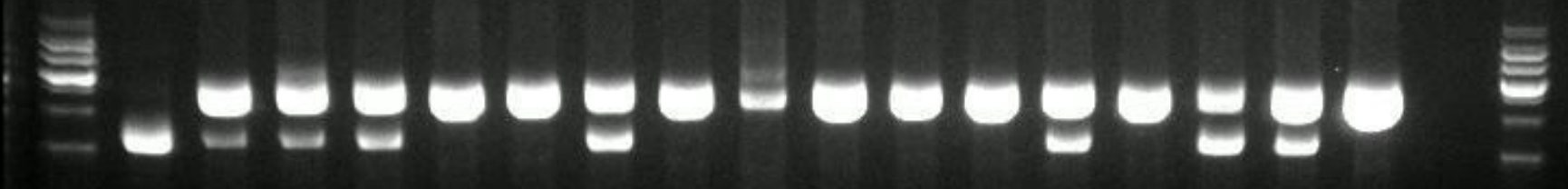
$-$ 


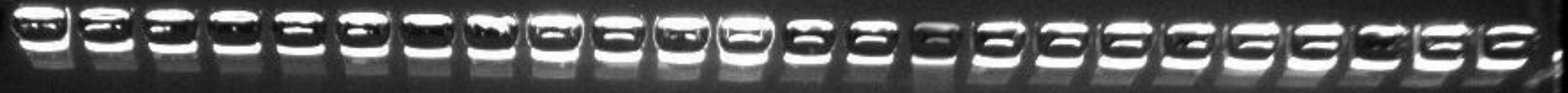

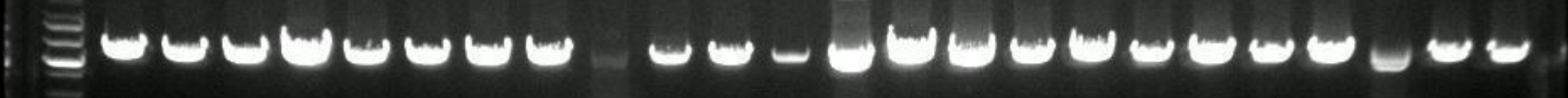

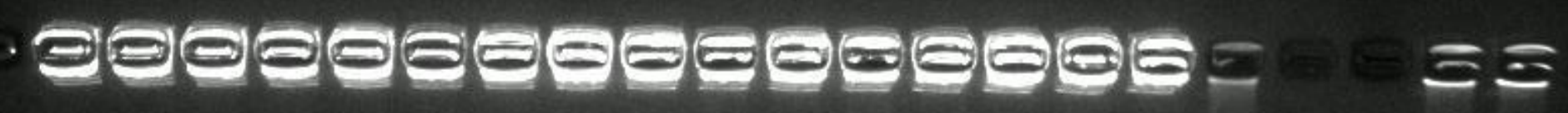

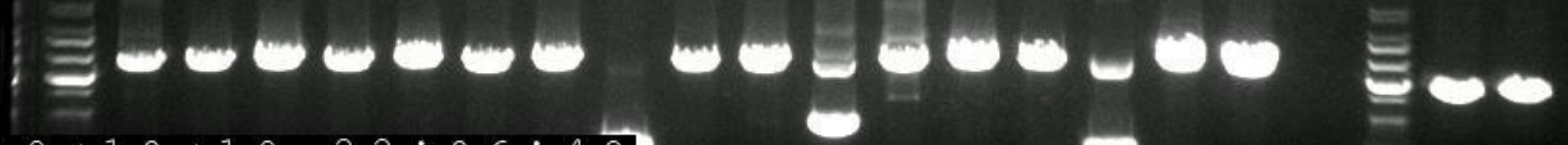

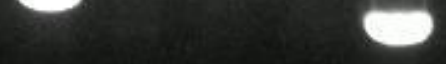




\section{Fig S22-d-2}

\begin{tabular}{|l|l|l|l|l|l|l|l|l|l|l|l|l|l|l|l|l|l|}
\hline M & 25 & 26 & 27 & 28 & 29 & 30 & 31 & 32 & 33 & 34 & 35 & 36 & 37 & 38 & 39 & 40 & 41 \\
\hline
\end{tabular}

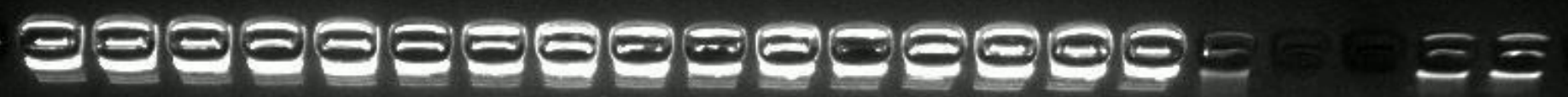

竎-000000 00 


\section{Fig S25-b-2}

\begin{tabular}{|l|l|l|l|l|l|l|l|l|l|l|l|l|}
\hline 25 & 26 & 27 & 28 & 29 & 30 & 31 & 32 & 33 & 34 & 35 & B & M \\
\hline
\end{tabular}

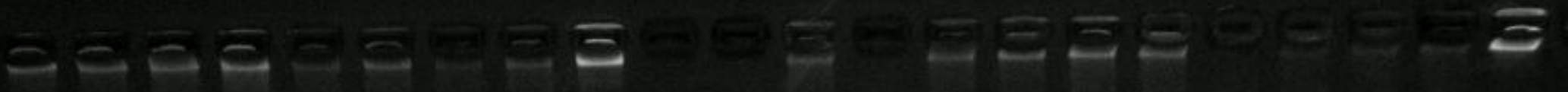

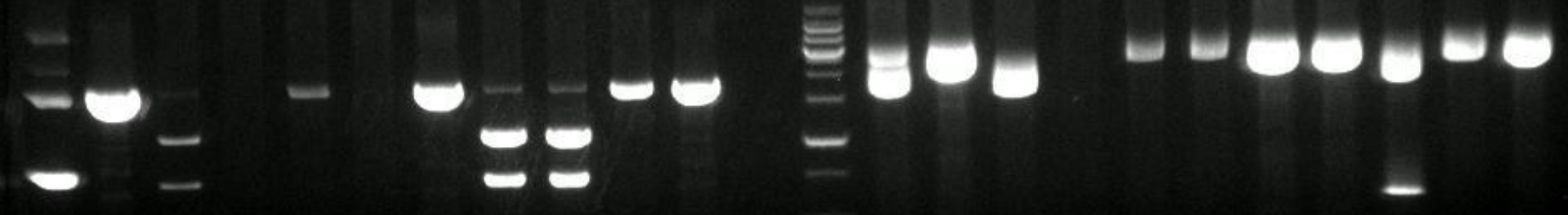




\section{Fig S25-b-3}

\begin{tabular}{|l|l|l|l|l|l|l|l|l|l|l|l|l|l|l|l|l|l|l|l|l|l|l|l|l|}
\hline $\mathrm{M}$ & 1 & 2 & 3 & 4 & 5 & 6 & 7 & 8 & 9 & 10 & 11 & 12 & 13 & 14 & 15 & 16 & 17 & 18 & 19 & 20 & 21 & 22 & 23 & 24 \\
\hline
\end{tabular}

르르트

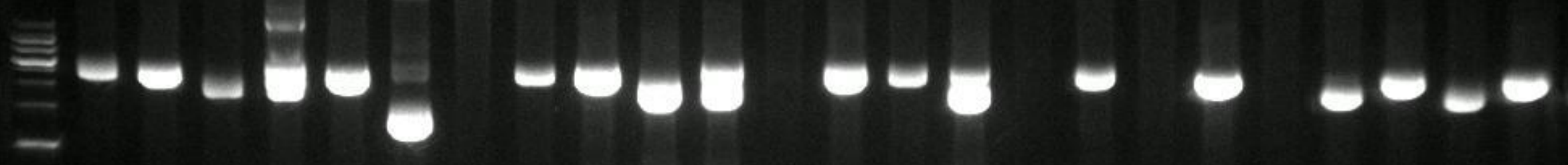

- -

$-$ 


\section{Fig S25-b-4}

\begin{tabular}{|l|l|l|l|l|l|l|l|l|l|l|l|l|}
\hline M & 25 & 26 & 27 & 28 & 29 & 30 & 31 & 32 & 33 & 34 & 35 & B \\
\hline
\end{tabular}

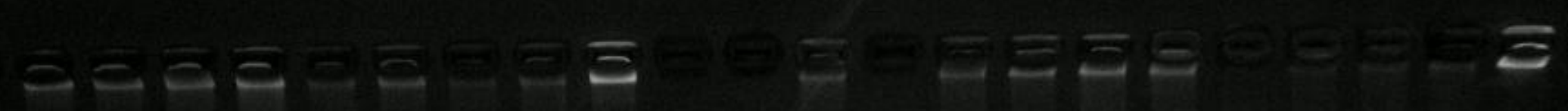

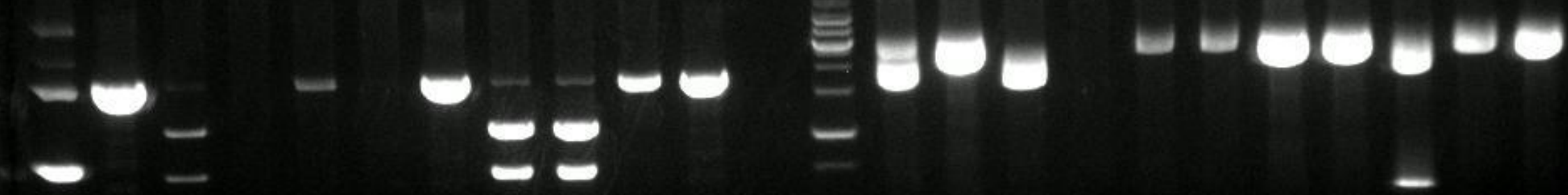


Fig S25-c

\begin{tabular}{|l|l|l|l|l|l|l|l|l|l|}
\hline M & $21-1$ & $21-0.2$ & $21-0.1$ & Wt & B & $14-1$ & $14-0.2$ & $14-0.1$ & M \\
\hline
\end{tabular}

붕

$\bullet$

븝 


\section{Fig S25-e-1}

\begin{tabular}{|l|l|l|l|l|l|l|l|l|l|l|l|l|l|l|l|l|l|l|l|l|l|l|l|l|}
\hline $\mathrm{M}$ & 1 & 2 & 3 & 4 & 5 & 6 & 7 & 8 & 9 & 10 & 11 & 12 & 13 & 14 & 15 & 16 & 17 & 18 & 19 & 20 & 21 & 22 & 23 & 24 \\
\hline
\end{tabular}

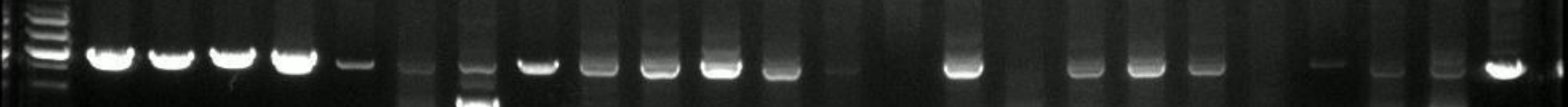
-............... 


\section{Fig S25-e-2}

\begin{tabular}{|l|l|l|l|l|l|l|l|l|l|l|l|}
\hline M & 25 & 26 & 27 & 28 & 29 & 30 & 31 & 32 & 33 & 34 & 35 \\
\hline
\end{tabular}

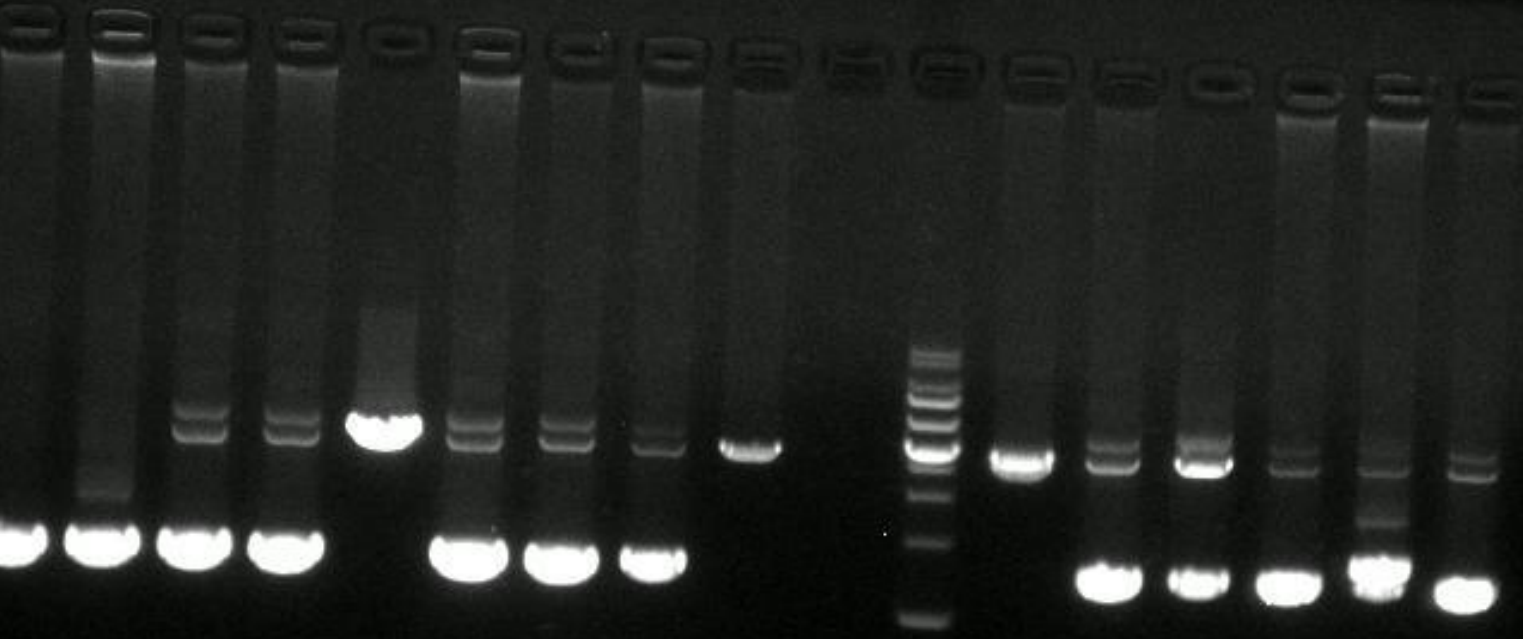

